\title{
A New Concept for Pacemaker Activity in the Human Stomach
}

(J Physiol 2011:589[Pt 24];6105-6118)

\section{Jeong Hwan Kim}

Department of Internal Medicine, Digestive Disease Center, Konkuk University School of Medicine, Konkuk University Medical Center, Seoul, Korea

\section{Summary}

Rhee et $\mathrm{al}^{1}$ has reported a study entitled "Analysis of pacemaker activity in the human stomach" in the December issue of Journal of Physiology 2011. Extracellular electrical recording techniques using animal models have contributed to establish important concepts of human gastric physiology. ${ }^{2,3}$ The electrical quiescence in the gastric fundus, 3 cycles per minute (cpm) pacemaker activity in corpus and antrum, and proximal-to-distal slow wave frequency gradient tended to accept as standard concepts. However, several findings which do not concur with those classic concepts have been presented in Rhee's study. Rhee et $\mathrm{al}^{1}$ experimented using human gastric muscles obtained from patients undergoing gastric resection for gastric cancer and investigated the slow wave pacemaker activity recorded with intracellular microelectrodes, the contractions recorded by isometric force techniques and the distribution of interstitial cells of Cajal (ICC).

Interestingly, different from the recent studies, slow waves were routinely recorded from gastric fundic muscles. And similar waveforms as slow waves in more distal regions were presented with couple to phasic contractions. Also gastric slow wave frequency was significantly greater than $3 \mathrm{cpm}$ in all regions of the stomach. Additionally antral slow wave frequency often exceeded the highest frequency of pacemaker activity in the corpus. These observations could not be explained as chronotropic mechanisms such as muscarinic and prostaglandin receptor binding, stretch, extracelluar $\mathrm{Ca}^{2+}$ and temperature. After all, muscles from all regions through the thickness of the muscularis demonstrated intrinsic pacemaker activity, and this corresponded with the widespread distribution in ICC.

\section{Comment}

The control of gastrointestinal movements by enteric motor neurons is critical for orderly processing of food, absorption of nutrients and elimination of wastes. Over the past several years, it has been suggested that motor neurotransmission is more complicated than simple release of neurotransmitters from nerve terminals and binding of receptors on smooth muscle cells. In fact, the 'neuro-effector' junction may consist of synaptic-like connectivity with specialized cells, and contributions from multiple cell types in integrated post-junctional responses. ICCs were proposed as potential mediators in motor neurotransmission based on reduced post-junctional responses observed in $W$ mutants that have reduced populations of ICC. ${ }^{4,5}$

Received: May 29, 2012 Revised: June 5, 2012 Accepted: June 8, 2012

(c) This is an Open Access article distributed under the terms of the Creative Commons Attribution Non-Commercial License (http://creativecommons. org/licenses/by-nc/3.0) which permits unrestricted non-commercial use, distribution, and reproduction in any medium, provided the original work is properly cited.

*Correspondence: Jeong Hwan Kim, MD

Department of Internal Medicine, Digestive Disease Center, Konkuk University School of Medicine, Konkuk University Medical Center, Neungdong-ro 120-1, Gwangjin-gu, Seoul 143-914, Korea

Financial support: None.

Tel: +82-2-2030-7450, Fax: +82-2-2030-7458, E-mail: sefamily@kuh.ac.kr

Conflicts of interest: None. 
Many of the principles of human gastric physiology and pathophysiology came from animal studies, most commonly using dog, guinea-pig and mouse. ${ }^{2,3}$ There have been relatively few studies of human gastric muscles, so it is uncertain whether the mechanisms and patterns of gastric activation, receptive relaxation and peristalsis in laboratory animals can be translated to human gastric physiology. Usually accepted standard concepts in human gastric electrophysiology include electrical quiescence in the fundus, a dominant slow wave frequency of $3 \mathrm{cpm}$, and an intrinsic slow wave frequency gradient where faster proximal pacemakers dominate over slower distal pacemakers. However, recently, there are several papers about human gastric muscles which reported that slow wave frequencies or phasic contractions were greater than that which has been considered normal for human gastric muscles (that is, above $4 \mathrm{cpm}$ in corpus, antrum, and even fundus) using intracellular recording. ${ }^{6,7}$

Extracellular recording from gastrointestinal muscles is prone to extensive motion artifact, and it is not clear that animal models can be translated directly to human physiology. Therefore, methodologically, this study performed a detailed analysis of electrical activity from carefully mapped specimens of gastric muscle removed from humans during surgery for gastric cancers. The data in this study showed several important differences in electrical activity recorded with intracellular microelectrodes and accepted gastric electrophysiological dogma; (1) the authors observed ongoing electrical slow wave activity greater than $3 \mathrm{cpm}$ in the gastric fundus as well as in the gastric antrum and corpus and (2) they also found no evidence for a slow wave frequency gra- dient. Muscles from all regions through the thickness of the muscularis demonstrated intrinsic pacemaker activity, and this corresponded with the widespread distribution of pacemaker cells. These findings suggest that extracellular electrical recording has underestimated human slow wave frequency and mechanisms of human gastric function may differ from standard laboratory animal models. Therefore, the $3 \mathrm{cpm}$ 'standard' for slow wave frequency, electrical fundic quiescence and slow wave frequency gradient need reconsideration.

\section{References}

1. Rhee PL, Lee JY, Son HJ, et al. Analysis of pacemaker activity in the human stomach. J Physiol 2011;589(Pt 24):6105-6118.

2. Lammers WJ, Ver Donck L, Stephen B, Smets D, Schuurkes JA. Origin and propagation of the slow wave in the canine stomach: the outlines of a gastric conduction system. Am J Physiol Gastrointest Liver Physiol 2009;296:G1200-G1210.

3. Kito Y, Suzuki H. Electrophysiological properties of gastric pacemaker potentials. J Smooth Muscle Res 2003;39:163-173.

4. Sanders KM, Hwang SJ, Ward SM. Neuroeffector apparatus in gastrointestinal smooth muscle organs. J Physiol 2010;588(Pt 23): 4621-4639.

5. Kim HJ. A functional role for the 'fibroblast-like cells' in gastrointestinal smooth muscles (J Physiol 2011;589[Pt 3]:697-710). J Neurogastroenterol Motil 2011;17:425-426.

6. el-Sharkawy TY, Morgan KG, Szurszewski JH. Intracellular electrical activity of canine and human gastric smooth muscle. J Physiol 1978;279;291-307.

7. Sinn DH, Min BH, Ko EJ, et al. Regional differences of the effects of acetylcholine in the human gastric circular muscle. Am J Physiol Gastrointest Liver Physiol 2010;299:G1198-G1203. 